\title{
What is Good for Hypertensive Patients: Presence or Absence of Presystolic Wave
}

\author{
Gulay Uzun ${ }^{1}$, Dilek KIRCI ${ }^{2}$, Levent Korkmaz ${ }^{3}$, Ali Akyüz ${ }^{4}$, and Muhammet Sayin ${ }^{5}$ \\ ${ }^{1}$ Affiliation not available \\ ${ }^{2}$ Trabzon Kanuni Egitim ve Arastirma Hastanesi \\ ${ }^{3}$ AHI EVREN HEARTAND VASCULAR SURGRY \\ ${ }^{4}$ Akcaabat Hackali Baba State Hospital \\ ${ }^{5}$ Bulent Ecevit University
}

October 1, 2020

\begin{abstract}
Background: The present study aimed at evaluating the feasibility of using presystolic wave as an indicator of early myocardial damage in patients with essential hypertension. Methods: The present study included 90 patients diagnosed with hypertension. The patients were divided into two groups on the basis of whether they exhibited the presence of PSW ( $\mathrm{n}=64$, age: $56 \pm 11$ years) or its absence $(\mathrm{n}=26$, age: $58 \pm 13$ years), as revealed by the results of left ventricular outflow Doppler echocardiography. The levels of Heart-type free fatty acid-binding protein were compared between the two groups. Results: In the 90 hypertensive patients included in the present study, the prevalence of presystolic wave was determined to be $71 \%$. The Heart-type free fatty acid-binding protein levels in the non-PSW patients were observed to be statistically higher than those in the PSW patients [4.88 ng/mL (2.82-17.83) vs. $3.99 \mathrm{ng} / \mathrm{mL}(2.57-17.87), \mathrm{p}=0.041]$. Even though the ejection fractions and the left ventricular diameters were within the normal limits for the patients of both the groups, the value for the left ventricular enddiastolic diameter was observed to be statistically higher in the non-PSW group. No differences were observed in the mitral flow parameters between the two groups. Conclusion: The present study demonstrated that the absence of presystolic wave in the hypertensive patients with preserved left ventricular ejection fraction is an indicator of subclinical myocardial damage. The hypertensive patients exhibiting the absence of the presystolic wave should be strictly followed up for cardiac complications.
\end{abstract}

\section{Introduction}

Left ventricular (LV) hypertrophy and remodeling, which are commonly observed in the hypertensive patients, arise as a result of the complex interaction of several hemodynamic and non-hemodynamic variables. These changes predispose to and exacerbate the impairment of left ventricular function, eventually leading to heart failure. ${ }^{1,2}$ In patients with hypertension (HT), it is crucial to identify the LV dysfunction as early as possible in order to identify the patients at high risk for heart failure.

Presystolic wave (PSW) is a late diastolic event that is often detected on the Doppler of the left ventricular outflow tract. ${ }^{3}$ Several reports assessing whether PSW is a normal phenomenon or a marker of pathological conditions are available in the literature. The clinical significance of the presence or absence of PSW in hypertensive patients has not been completely established so far.

Heart-type free fatty acid-binding protein (H-FABP) is a cytosolic protein that is released rapidly from the myocardium in response to tissue damage and may be utilized as a marker for myocardial damage. ${ }^{4}$ It is well known that hypertensive patients exhibit elevated levels of H-FABP compared to normotensive patients. ${ }^{5}$

The aim of the present study was to evaluate the feasibility of using the presystolic wave detected in the 
Doppler echocardiography of the LV outflow tract in patients with essential HT as a marker for early myocardial damage through comparison with the H-FABP levels.

\section{Methods}

The present study included 90 patients who had applied to the Cardiology and Internal Diseases outpatient clinics, ... (blinded), during the period between March 2017 and May 2017, and were diagnosed with HT [systolic blood pressure (SBP) [?] $140 \mathrm{mmHg}$ and/or diastolic blood pressure (DBP) [?] $90 \mathrm{mmHg}$ or those currently receiving treatment] based on the diagnosis criteria provided by the European Society of Cardiology. The patients were divided into two groups on the basis of whether they exhibited the presence of the PSW ( $\mathrm{n}=64$, age: $56 \pm 11$ years) or its absence $(\mathrm{n}=26$, age: $58 \pm 13$ years), as detected in the Doppler echocardiography of the LV outflow. The exclusion criteria for the present study were as follows: secondary HT, hypertrophic obstructive cardiomyopathy, severe renal and hepatic dysfunction, LV systolic dysfunction, heart valve disease, coronary artery disease, a history of myocardial infarction, congenital heart disease, atrial fibrillation, any degree of atrioventricular block, severe bradycardia, sinus syndrome, having a pacemaker, presence of complete bundle branch block, electrolyte disorder, any kind of malignancy, pregnancy, asthma or advanced chronic obstructive pulmonary disease, aortic diseases, connective tissue disorders, and use of additional medication that affects blood pressure.

The present study was approved by the ethics committee of Trabzon Kanuni Training and Research Hospital. Both oral and written forms of consent were obtained from the patients after they had been informed thoroughly regarding the study.

\section{Measurements}

The clinical and demographic evaluations and the echocardiographic and blood pressure measurements of all the patients were performed by the same investigator inside one particular room during the morning hours.

Overall assessment and blood pressure measurements

Medical histories of all the patients were collected, following which each patient underwent a physical examination. Normal findings were obtained for all the subjects upon physical examination. Teleradiograms and electrocardiograms were observed to be normal for all patients. In order to calculate the aortic elastic parameters, SBP and DBP were measured simultaneously using a mercury sphygmomanometer during the echocardiographic examination. In addition, heart rate was recorded simultaneously using an electrocardiograph.

Echocardiographic assessment

The standard echocardiographic procedures were performed using a Vivid System 5 device (General Electric, Horten, Norway). A 2.5-MHz probe was utilized for the Doppler measurements. The measurements were performed in accordance with the guidelines provided by the American Society of Echocardiography and were reported as the average of three cardiac cycles(6). The LV dimensions and wall thickness were obtained from the parasternal long axis with an M-mode cursor positioned just beyond the tips of the mitral leaflet, perpendicular to the long axis of the ventricle. LV end-diastolic diameter (LVEDD) and LV end-systolic (LVESD) diameter were measured.

Mitral inflow velocities were evaluated using the pulsed-wave the Doppler, with sample volume placed at the tip of the mitral leaflets in an apical four-chamber view. Using the average of three beats, the diastolic peak early (E) and peak late (A) transmitral flow velocities and the peak E to peak A velocities (E/A) were measured. LV ejection fraction(LVEF) was estimated using the Simpson's method.

PSW was assessed in an apical five-chamber view, using the pulsed-wave Doppler echocardiography, with the Doppler beam aligned parallel to the direction of the LV outflow tract(LVOT) flow and the sample volume approximately $1 \mathrm{~cm}$ above the aortic valve leaflet tips. PSW peak velocities were obtained only in the event of a PSW signal. Therefore, the PSW peak velocities were recorded and analyzed only in the patients exhibiting the presence of PSW. 


\section{H-FABP measurement}

Biochemistry tubes were filled with 5 cc volume of peripheral venous blood samples obtained from the patients. The samples were centrifuged at 2,000 rpm for $15 \mathrm{~min}$ and serum samples were obtained, which were stored at $-80 \operatorname{deg} \mathrm{C}$ until the time of analysis. After all the samples were obtained from the patients, the levels of serum H-FABP were determined using the solid-phase ELISA (Enzyme Linked-Immuno-Sorbent Assay) method with ready-to-use ELISA kits (Sunredbio, China).

\section{Statistical Analysis}

Statistical analysis of the results of the present study was performed using the SPSS for Windows Version 19 (SPSS Inc., Chicago, Illinois, USA). Continuous variables were expressed as mean +- standard deviation or median (minimum-maximum), while the categorical variables were expressed as numbers and percentages. The suitability of the data for normal distribution was evaluated using the Kolmogorov-Smirnov test. The categorical variables were evaluated using the Chi-Squared Test. In the case of continuous variables, independent groups t-test was utilized for the data suitable for normal distribution, while the Mann-Whitney U-test was utilized for the data not suitable for normal distribution. Statistical significance was accepted at a p-value of $<0.05$ for all the statistical analyses.

\section{Results}

The prevalence of presystolic wave in the 90 hypertensive patients included in the present study was determined to be $71 \%$. The patients were divided into two groups on the basis of the presence or absence of PSW (PSW group, $\mathrm{n}=64$, age: $56+-11$ years; non PSW group, $\mathrm{n}=26$, age: $58+-13$ years). The clinical and demographic characteristics of the patients have been listed in Table 1. There were no differences between the two groups of patients in terms of the drugs used by these patients (Table 2). The levels of H-FABP were observed to be statistically higher in the non-PSW patients compared to the PSW patients $[4.88 \mathrm{ng} / \mathrm{mL}$ $(2.82-17.83)$ vs. $3.99 \mathrm{ng} / \mathrm{mL}(2.57-17.87), \mathrm{p}=0.041]$ (Table 3). Even though the ejection fractions and the LV diameters were determined to be within the normal limits for the patients in both the groups, the LVEDD was observed to be statistically higher in the non PSW group. No differences in the mitral flow parameters were observed between the groups (Table 4).

\section{Discussion}

The ejection fraction was preserved, and the FABP levels, which serve as a marker for myocardial damage, were observed to be statistically higher in the non-PSW hypertensive patients compared to the PSW patients.

PSW is a late diastolic event that is often observed in the Doppler analysis of the LVOT $^{3}$ Various previous studies have reported different prevalence values for PSW. The prevalence of PSW varied with the studied population and has been reported to vary between $64 \%$ and $95 \% .^{7,8}$ In the present study, the prevalence of PSW in the hypertensive patient population was determined to be $71 \%$. Different views have been reported regarding whether PSW is a normal phenomenon or a marker for pathological conditions. It has been suggested that it occurs as a result of atrial contractions against a typically hypertrophied ventricle exhibiting increased stiffness and increased diastolic pressure. However, it may also be present in normal individuals and may produce different projections under different clinical situations. In a study conducted by Akyuz et al., the left ventricular wall thickness was observed to be more hypertrophic in the group of hypertensive patients with PSW compared to the non-PSW group. ${ }^{9}$ In the present study, no difference in terms of wall thickness was observed between the groups. Again, in the study conducted by Akyuz et al., LVDD was detected in the PSW group in contrast to the non-PSW group. ${ }^{9}$ In another study conducted by Mittal et al., PSW was demonstrated to be associated with LVDD. ${ }^{10}$ In the present study, LVDD was detected in the patients belonging to both PSW and non-PSW groups. The presence or absence of PSW did not result in any difference in terms of diastolic dysfunction. In a study conducted by Dursun et al., the presence of PSW detected in the Doppler echocardiography of the patients with acute myocardial infarction was observed to be associated with higher LV ejection fraction and lower grade diastolic dysfunction. ${ }^{11} \mathrm{~A}$ study conducted by Panayiotou et al. reported diastolic mitral flow wave velocity and LVEDD as the main 
factors affecting the presystolic wave velocity. ${ }^{8}$ In the present study, the left ventricular end-diastolic diameter was observed to exhibit a statistical increase in the PSW group compared to the non PSW. All these findings suggested that PSW has a biphasic response. While the prominence of the diastolic dysfunction increases due to the strengthening of the wave in the early period, the PSW distinction decreases and eventually disappears as the diastolic dysfunction progresses and the systolic functions begin to be affected.

Previous studies have demonstrated that myocyte damage occurs in the early stages of hypertensive heart disease. ${ }^{12,13} \mathrm{H}-\mathrm{FABP}$ is a cytosolic protein with considerably low molecular weight, which is released rapidly from the myocardium in response to tissue damage. ${ }^{4}$ In a study conducted by Gedikli et al., H-FABP levels representing myocardial damage were observed to be higher in the hypertensive patients compared to the normotensive patients. ${ }^{5}$ In the present study, the H-FABP levels were observed to be higher in the non PSW HT patients compared to the PSW group patients. This finding suggested that the absence of PSW in hypertensive patients might be associated with early myocardial damage. In the study conducted by Joshi et al., the absence of PSW, particularly in patients with low ejection fraction, was observed to be associated with increased cardiovascular outcomes. This corroborates the conclusion that the absence of PSW is associated with increased myocardial damage.

\section{Conclusion}

Convenient-to-use parameters that would be able to predict the transition to systolic dysfunction are required for the treatment of hypertensive patients. The present study demonstrated that the absence of PSW in hypertensive patients with preserved LV ejection fraction is an indicator of subclinical myocardial damage. The non-PSW hypertensive patients should be strictly followed up for cardiac complications.

\section{Acknowledgements}

We thank all participants for their cooperation.

\section{Funding Sources}

This investigation did not receive any specific grant from funding agencies in the public, commercial, or not-for-profit sectors

\section{Disclosures}

The authors have no conflicts of interest to disclose.

\section{Declarations of interest}

None.

Table 1. Clinical and demographic characteristics of patients.

\begin{tabular}{|c|c|c|c|}
\hline & Patients with PSW $n=64$ & $\begin{array}{l}\text { Patients without PSW } \\
n=26\end{array}$ & $\mathrm{P}$ \\
\hline Age (years) & $56 \pm 11$ & $58 \pm 13$ & 0.443 \\
\hline Female Sex (n, \%) & $15(57 \%)$ & $53(82 \%)$ & 0.012 \\
\hline Heart Rate (beat/min) & $75 \pm 12$ & $72 \pm 13$ & 0.271 \\
\hline $\begin{array}{l}\text { Systolic Blood Pressure } \\
(\mathrm{mmHg})\end{array}$ & $130(120-150)$ & $130(120-180)$ & 0.398 \\
\hline $\begin{array}{l}\text { Diastolic Blood } \\
\text { Pressure }(\mathrm{mmHg})\end{array}$ & $80(60-100)$ & $80(70-95)$ & 0.238 \\
\hline $\begin{array}{l}\text { Hypertension Period } \\
\text { (months) }\end{array}$ & $60(1.2-480)$ & $60(1.2-360)$ & 0.731 \\
\hline $\begin{array}{l}\text { Family History of } \\
\text { Coranary Artery } \\
\text { Disease (n, \%) }\end{array}$ & $21(32 \%)$ & $5(19 \%)$ & 0.198 \\
\hline
\end{tabular}




\begin{tabular}{llll}
\hline & Patients with PSW n=64 & $\begin{array}{l}\text { Patients without PSW } \\
\mathrm{n}=26\end{array}$ & $\mathrm{P}$ \\
\hline Smoking (n, \%) & $8(12 \%)$ & $2(\% 8)$ & 0.511 \\
$\begin{array}{l}\text { Diabetes Mellitus (n, } \\
\text { \%) }\end{array}$ & $19(29 \%)$ & $4(15 \%)$ & 0.159 \\
Hyperlipidemia (n, \%) & $18(28 \%)$ & $5(19 \%)$ & 0.381 \\
$\begin{array}{l}\text { Body Mass Index } \\
\left(\mathrm{kg} / \mathrm{m}^{2}\right)\end{array}$ & $33.2 \pm 5.6$ & $31.1 \pm 5.8$ & 0.129 \\
\hline
\end{tabular}

Table 2. Drugs used by patients.

\begin{tabular}{|c|c|c|c|}
\hline & Patients with PSW n=64 & $\begin{array}{l}\text { Patients without PSW } \\
\mathrm{n}=26\end{array}$ & $\mathrm{P}$ \\
\hline $\operatorname{ACEI}(\mathrm{n}, \%)$ & $14(22 \%)$ & $9(34 \%)$ & 0.209 \\
\hline $\operatorname{ARB}(n, \%)$ & $21(32 \%)$ & $7(27 \%)$ & 0.584 \\
\hline Thiazide $(\mathrm{n}, \%)$ & $4(6 \%)$ & $0(0 \%)$ & 0.192 \\
\hline Beta Blocker (n, \%) & $14(22 \%)$ & $3(11 \%)$ & 0.256 \\
\hline $\mathrm{CCB}(\mathrm{n}, \%)$ & $12(19 \%)$ & $8(30 \%)$ & 0.214 \\
\hline Alpha Blocker (n, \%) & $2(3 \%)$ & $0(0 \%)$ & 0.362 \\
\hline Spironolactone (n, \%) & $1(2 \%)$ & $0(0 \%)$ & 0.522 \\
\hline Statin $(n, \%)$ & $4(6 \%)$ & $0(0 \%)$ & 0.192 \\
\hline
\end{tabular}

ACEI: Angiotensin-converting enzyme inhibitor, ARB: Angiotensin II receptor blocker, CCB: Calcium channel blocker.

Table 3. H-FABP and biochemical values.

\begin{tabular}{llll}
\hline & & Patients without PSW \\
& Patients with PSW $\mathrm{n}=64$ & $\mathrm{n}=26$ & $\mathrm{P}$ \\
\hline H-FABP $(\mathrm{ng} / \mathrm{mL})$ & $3.99(2.57-17.87)$ & $4.88(2.82-17.83)$ & 0.041 \\
Glucose $(\mathrm{mg} / \mathrm{dL})$ & $107.5(76-241)$ & $101.5(76-393)$ & 0.769 \\
CRP $(\mathrm{mg} / \mathrm{dL})$ & $0.35(0.0-8.5)$ & $0.5(0.1-2.6)$ & 0.444 \\
UREA $(\mathrm{mg} / \mathrm{dL})$ & $30.2 \pm 8.5$ & $30.8 \pm 6.7$ & 0.743 \\
CREATINE (mg/dL) & $0.74 \pm 0.12$ & $0.75 \pm 0.18$ & 0.743 \\
eGFR (mL/min/1.73 & $94.5 \pm 12.1$ & $95.4 \pm 17.1$ & 0.776 \\
$\left.\mathrm{~m}^{2}\right)$ & & & \\
LDL $(\mathrm{mg} / \mathrm{dL})$ & $159 \pm 33$ & $149 \pm 26$ & 0.226 \\
HDL $(\mathrm{mg} / \mathrm{dL})$ & $48 \pm 10$ & $48 \pm 11$ & 0.912 \\
Triglyceride $(\mathrm{mg} / \mathrm{dL})$ & $164 \pm 56$ & $162 \pm 83$ & 0.891 \\
NA (mmol/L) & $139 \pm 2.1$ & $139 \pm 2.6$ & 0.812 \\
K (mmol/L) & $4.3 \pm 0.4$ & $4.4 \pm 0.3$ & 0.183 \\
\hline
\end{tabular}

CRP: C Reactive Protein; eGFR: Estimated Glomerular Filtration Rate; H-FABP: Heart-type free fatty acid-binding protein; LDL: low-density lipoprotein cholesterol; HDL: High density lipoprotein cholesterol

Table 4. Echocardiographic parameters. 


\begin{tabular}{llll}
\hline & & Patients without PSW \\
$\mathrm{n}=26$ & $\mathrm{p}$ \\
\hline EF $(\%)$ & Patients with PSW n=64 & $65(55-70)$ & 0.895 \\
LA (mm) & $34.8 \pm 3.6$ & $36.2 \pm 3.1$ & 0.081 \\
IVS (mm) & $10(7-14)$ & $10(8-14)$ & 0.239 \\
PW (mm) & $10(7-14)$ & $10(8-13)$ & 0.282 \\
LVEDD (mm) & $46.6 \pm 4.5$ & $49.3 \pm 3.5$ & 0.008 \\
LVESD (mm) & $29.3 \pm 5.5$ & $31.6 \pm 4.2$ & 0.065 \\
Mitral E (cm/sec) & $65.9 \pm 17.4$ & $66.5 \pm 13.3$ & 0.0881 \\
Mitral A (cm/sec) & $76.6 \pm 18.6$ & $79.3 \pm 20.7$ & 0.547 \\
E/A & $0.78(0.48-2)$ & $0.77(0.52-1.9)$ & 0.940 \\
\hline
\end{tabular}

EF:Ejection fraction; IVS: Interventricular septum; LA: Left atrium; LVEDD: Left ventriculer end-diastolic diameter; LVESD: Left ventricular end-sistolic diameter; PSW: Presystolic wave; PW:Posterior Wall

\section{References}

1. Frohlich ED, Apstein C, Chobanian AV, et al. The heart in hypertension. N Engl J Med 1992; 327:9981008 .

2. Lorell BH, Carabello BA. Left ventricular hypertrophy: pathogenesis, detection, and prognosis. Circulation 2000; 102:470-479.

3. Armstrong WF, Ryan T, Feigenbaum H. Feigenbaum's echocardiography. Philadelphia: Wolters Kluwer Health/Lippincott Williams \& Wilkins; 2010:545.

4. Glatz JFC, Van Bilsen M, Paulussen RJA, et al. Release of fatty acid-binding protein from isolated rat heart subjected to ischemia and reperfusion or to the calcium paradox. Biochim Biophys Acta1988;961:14852.

5. Gedikli O, Ozturk S, Yilmaz H, et al. Relationship between arterial stiffness and myocardial damage in patients with newly diagnosed essential hypertension. Am J Hypertens 2008;21 (9):989-93.

6. Quiñones MA, Otto CM, Stoddard M, et al, Doppler Quantification Task Force of the Nomenclature and Standards Committee of the American Society of Echocardiography. Recommendations for quantification of Doppler echocardiography: a report from the Doppler Quantification Task Force of the Nomenclature and Standards Committee of the American Society of Echocardiography. J Am Soc Echocardiogr 2002;15:167-84.

7. Joshi KR, Kabirdas D, Romero-Corral A, et al. Clinical significance of a presystolic wave on the Doppler examination of the left ventricular outflow tract. Am J Cardiol 2014,15;114:1599-602.

8. Panayiotou H, Byrd BF III. Origin and significance of diastolic Doppler flow signal in the left ventricular outflow tract. J Am Coll Cardiol, 1990;16:1625-1631.

9. Akyüz AR, Turan T, Gürbak I, et al. The relationship between presystolic wave and subclinical left ventricular dysfunction in asymptomatic hypertensive patients. Blood Press Monit. 2016;21 (5):277-81.

10. Mittal SR, Pancholi N. Left ventricular outflow tract presystolic flow velocity-another marker of left ventricular diastolic function. Int $J$ Cardiovasc Imaging 2002;18 (4):249-56.

11. Dursun, I, Kul S, Sahin S, et al. Relation of presystolic wave on doppler examination to syntax score in patients with acute myocardial infarction. Int J Cardiovasc Imaging 2017; 34 (4), 569-576.

12. Gonzalez A, Ravassa S, Lopez B, et al. Apoptosis in hypertensive heart disease: a clinical approach. Curr Opin Cardiol 2006; 21: 288-294. 
13. Unlu M, Temiz NH, Cengel A. Myocardial indium-111-antimyosin uptake in essential hypertension. Nuklearmedizin 2003; 42:99-103. 